University of Wollongong

Research Online

Faculty of Engineering - Papers (Archive)

Faculty of Engineering and Information

Sciences

June 2003

\title{
Magnetospectroscopy of Be in GaAs
}

R. A. Lewis

University of Wollongong, roger@uow.edu.au

Y. Wang

National High Magnetic Field Laboratory, Tallahassee, USA

M. Henini

University of Nottingham, UK

Follow this and additional works at: https://ro.uow.edu.au/engpapers

Part of the Engineering Commons

https://ro.uow.edu.au/engpapers/234

\section{Recommended Citation}

Lewis, R. A.; Wang, Y.; and Henini, M.: Magnetospectroscopy of Be in GaAs 2003.

https://ro.uow.edu.au/engpapers/234

Research Online is the open access institutional repository for the University of Wollongong. For further information contact the UOW Library: research-pubs@uow.edu.au 


\title{
Magnetospectroscopy of Be in GaAs
}

\author{
R. A. Lewis* \\ Institute for Superconducting and Electronic Materials, University of Wollongong, Wollongong NSW 2522, Australia \\ Y.-J. Wang \\ National High Magnetic Field Laboratory, Tallahassee, Florida 32310
}

\section{Henini}

School of Physics and Astronomy, University of Nottingham, Nottingham NG7 2RD, United Kingdom

(Received 31 August 2002; revised manuscript received 23 December 2002; published 19 June 2003)

\begin{abstract}
Far-infrared absorption measurements have been made of the Lyman series of the acceptor Be in GaAs up to magnetic fields of $30 \mathrm{~T}$, supplemented by photothermal ionization spectroscopy measurements to $6 \mathrm{~T}$. The results confirm and greatly extend previous work and reveal several phenomena. The strongest component of the $G$ line moves to higher energies and increases in intensity with field. Around $20 \mathrm{~T}$ the field dependence of the transition displays a marked kink. At higher fields the dependence of energy on field is linear. This behavior is attributed to unresolved components that change in relative intensity with field and leads to a reappraisal of the identity of the low-field transition. The $D$ line is followed to high fields allowing an accurate determination of the splitting of the field-induced components of the second excited state. Photothermal ionization spectra for the $C$ line permit the determination of the splitting of the field-induced components of the ground state and of the third excited state. At high magnetic field an absorption appears which increases in energy with field at a much greater rate than any of the lines mentioned above. It is thought to originate in the valence-band Landau levels.
\end{abstract}

DOI: 10.1103/PhysRevB.67.235204

PACS number(s): 75.47.Gk, 78.30.-j, 63.20.Dj

\section{INTRODUCTION}

The intentional introduction of foreign atoms into highquality semiconductor crystals is the basis of much of the electronic technology that underpins the modern information economy. Impurity atoms may introduce energy states just above (below) the valence (conduction) band of the host. Such shallow levels have been the subject of intense study from both theoretical and experimental vantage points. The research has been reviewed several times ${ }^{1,2}$ and new developments are continually reported in a series of specialist conferences. ${ }^{3}$

We now focus on shallow impurities in a specific III-V compound semiconductor, GaAs. The conduction band of $\mathrm{GaAs}$ is almost parabolic and isotropic with the band minimum at the zone center. As a result the energy levels of a donor in GaAs closely match the energy levels of atomic hydrogen scaled by the factors of the electron effective mass $m^{*}$ and the dielectric constant of the lattice $\epsilon$ with an effective Rydberg

$$
R y^{*}=\frac{m^{*}}{m_{e}} \frac{1}{\epsilon^{2}} R y .
$$

In a magnetic field the degeneracy of the impurity energy states is lifted, analogously to the Zeeman effect in atomic hydrogen. Magnetospectroscopy measurements of transitions between magnetic-field split impurity states permit the precise determination of the electron effective mass. ${ }^{4}$ The application of higher magnetic fields allows the observation of small variations from the simple hydrogenic model, such as the nonparabolicity and anisotropy of the conduction band ${ }^{5}$ or the dependence of the energy of the ground state on the chemical identity of the donor. ${ }^{6}$ A dimensionless measure of magnetic field,

$$
\gamma=\frac{\hbar \omega}{2 R y}
$$

is useful in comparing hydrogen-like systems. For donors in GaAs, $\gamma=1$ corresponds to $6.57 \mathrm{~T}$; for the hydrogen atom, $\gamma=1$ corresponds to $2.35 \times 10^{5} \mathrm{~T}$.

Acceptors in GaAs may also be described using a model based on atomic hydrogen, but the problem is more complicated than for donors in view of the relatively complex structure of the valence band, characterized by the Luttinger parameters $\gamma_{1}, \gamma_{2}$, and $\gamma_{3}$. The energies are not given by so simple a relation as implied in Eq. (1); the calculation of acceptor energy levels will be described in the following section. An appropriate measure for the strength of the magnetic field is

$$
\beta_{0}=\frac{R y^{*}}{\gamma_{1} \mu_{B}},
$$

where $\mu_{B}$ is the Bohr magneton. For acceptors in GaAs, $\beta_{0}=34 \mathrm{~T}$. In small magnetic fields, $B \leqslant 7 \mathrm{~T}$, corresponding to $\beta \ll 1$, spectra have been reported for $\mathrm{C}$, $\mathrm{Zn}$, and $\mathrm{Be}$ acceptors in GaAs. ${ }^{7-9}$ One report has been given of acceptor spectra in intermediate magnetic fields: ${ }^{10} B$ to $17.5 \mathrm{~T}, \beta$ to $\approx 1 / 2$. The present work extends the magnetic-field range to high magnetic fields: $B=30 \mathrm{~T}, \beta \approx 1$. 


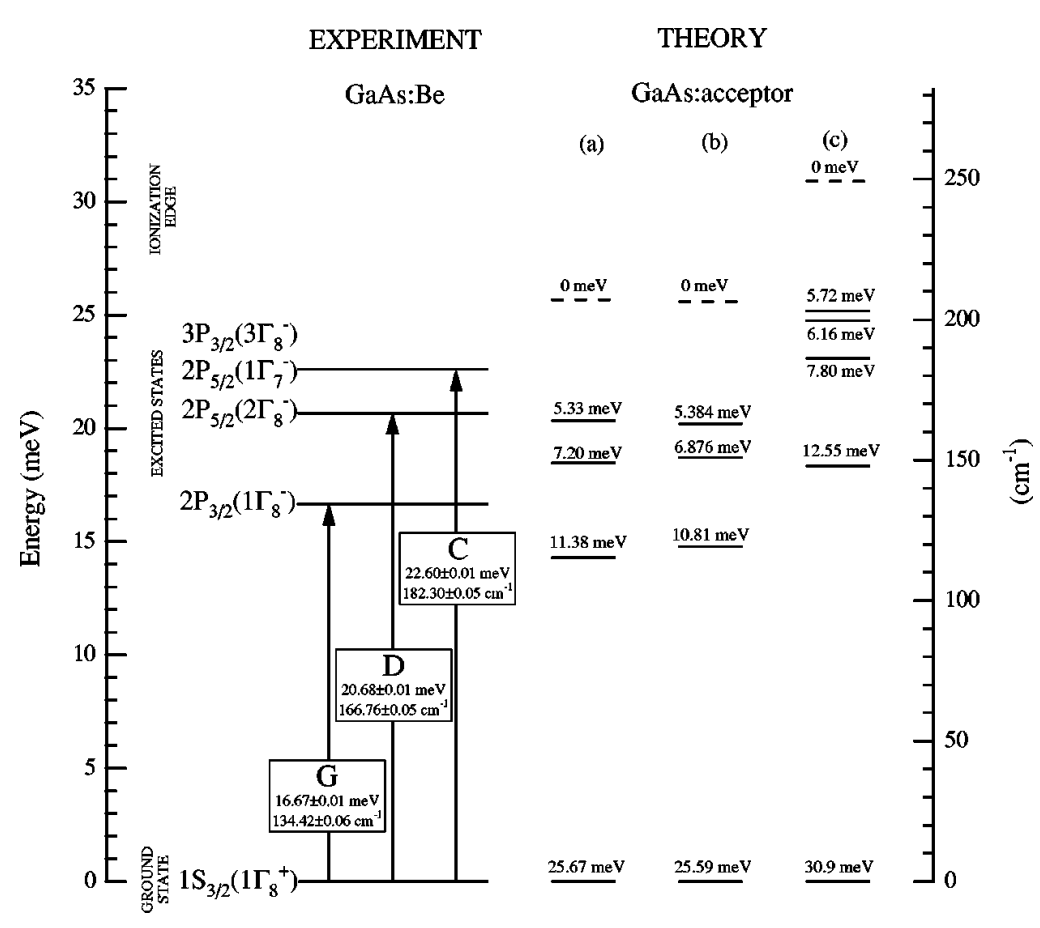

FIG. 1. Some energy states and optical transitions for acceptors in GaAs. The experimental data is for the Be acceptor (Ref. 17). The observed optical transitions are from the (evenparity) ground state to the lowest-energy oddparity states. The calculated data are from (a) Ref. 12, (b) Ref. 14, and (c) Ref. 13, respectively. The calculated ground-state energies have been aligned with the experimental zero of energy for ease of comparison. Note that there is considerable variation among the calculations, and between the calculations and experiment, regarding the separation of the even-parity and odd-parity states, whereas the separations of the odd-parity states are in much better agreement.

\section{THEORY}

\section{A. Unperturbed energy states}

A substitutional acceptor is located at a site of tetrahedral symmetry. In spite of this, Baldereschi and Lipari ${ }^{11}$ introduced a model which gives insight into the acceptor states and a tractable computational technique by beginning with states of spherical symmetry. This approach is viable in that the cubic terms in the Hamiltonian are small and so can be regarded as a perturbation. ${ }^{12}$ In a zinc blende, as opposed to a diamond, structure a term linear in wave vector appears in the Hamiltonian, but this is known from experiment to be small and is generally ignored in calculations.

The inclusion of the spin-orbit coupling in the spherical model requires atomic $S$ states to be denoted by $S_{3 / 2}$, where the subscript gives the total angular momentum $F$. The $P$ states become $P_{1 / 2}, P_{3 / 2}$, and $P_{5 / 2}$. Within this model, the energies of the $2 P_{1 / 2}, 2 P_{3 / 2}$, and $2 P_{5 / 2}$ states for GaAs have been calculated to be $1.6,11.1$, and $6.5 \mathrm{meV}$, respectively. ${ }^{11}$

Of the states so far discussed, adding first-order cubic terms to the Hamiltonian ${ }^{12}$ only affects the $2 P_{5 / 2}$ state, which now separates into $2 P_{5 / 2}\left(\Gamma_{8}^{-}\right)$and $2 P_{5 / 2}\left(\Gamma_{7}^{-}\right)$, where the terms $\Gamma_{k}^{\pi}$ refer to irreducible representations of the $O_{h}$ group. Further refinements to this spherical/cubic model discussed below do not introduce new states and so it is sufficient to label a state as $n \Gamma_{k}^{\pi}$ of $O_{h}$, where $\pi$ is the parity, denoted by + or,$- k$ takes on the values 6,7 , or 8 , and $n$ $=1,2,3, \ldots$ is the number of the state of a given $\pi$ and $k$ in order of energy. While the symbol $n \Gamma_{k}^{\pi}$ completely specifies the state, it is convenient to retain the atomic notation. Thus, the ground state will be written as $1 S_{3 / 2}\left(1 \Gamma_{8}^{+}\right)$and the first excited state as $2 P_{3 / 2}\left(1 \Gamma_{8}^{-}\right)$; these appear in the literature as $1 S_{3 / 2}\left(\Gamma_{8}^{+}\right)$and $2 P_{3 / 2}\left(\Gamma_{8}^{-}\right)$, respectively.
The energies of the ground and excited states of generic acceptors in GaAs have been calculated by Baldereschi and Lipari ${ }^{11}$ using the spherical Hamiltonian, and also incorporating the cubic and other terms, ${ }^{12}$ by Fiorentini, ${ }^{13}$ and by Schmitt et al. ${ }^{14}$ The most detailed results are given by the latter authors who present energy levels for 60 acceptor states. The energies these authors determine for acceptor states in GaAs relevant to the present experiments are illustrated in Fig. 1.

\section{B. Energy states in a magnetic field}

The theory of the Zeeman effect on acceptor states has been developed from the perspective of group theory by Bhattacharjee and Rodriguez. ${ }^{15}$

The experiments described below were carried out with the magnetic field parallel to the [001] crystal axis. In this geometry, the energies of the four sublevels of a $\Gamma_{8}$ state are given by

$$
E_{m}^{p}=\mu_{B}\left(m g_{1}^{p}+m^{3} g_{2}^{p}\right) B+\left[q_{1}^{p}+\left(q_{2}^{p}+q_{3}^{p}\right) m^{2}\right] B^{2},
$$

where $m= \pm \frac{1}{2}, \pm \frac{3}{2}$ is a spin quantum number characterizing the state, here given within the $T_{d}$ notation, the terms $g$ and $q$ are parameters of the material, and the label $p$ denotes the particular $\Gamma_{8}$ state. This equation is pertinent to the $1 S_{3 / 2}\left(1 \Gamma_{8}^{+}\right), 2 P_{3 / 2}\left(1 \Gamma_{8}^{-}\right)$, and $2 P_{5 / 2}\left(2 \Gamma_{8}^{-}\right)$states to be discussed below, for which the labels $p={ }^{\prime}, G$, and $D$, respectively, will be used.

Likewise, the energies of the two sublevels of a $\Gamma_{7}$ state are given by

$$
E_{m}^{p}=\mu_{B} m g^{p} B+q^{p} B^{2} .
$$

This equation is pertinent to the $2 P_{5 / 2}\left(1 \Gamma_{7}^{-}\right)$state to be discussed below, for which the label $p=C$ will be used. 
While strictly applicable only in the case when the magnetic-field induced splitting is small compared to the separation between unperturbed states and to the separation between the unperturbed states and other bands, in practice the theory can be applied over a large range of magnetic fields.

A detailed numerical theory of the Zeeman effect on acceptor states, including those in GaAs, has been presented by Schmitt et al. ${ }^{14}$ The calculation takes into account contributions of states with high angular momentum to improve accuracy. These authors present in detail the behavior of the ground state and the first five odd-parity excited states over the field range $0-10 \mathrm{~T}$ and give $g$ factors for the ground state and for the first two odd-parity excited states. The authors remark that the energies of the sublevels are adequately represented by the above equations of Bhattacharjee and Rodriguez only to about one-fifth of $\beta_{0}$ which, in the case of GaAs, is about 7 T. While not disputing that this may be true of the energy sublevels themselves, the determination of the energy splitting of the sublevels from the experimental data obtained by observing transitions between states often reveals a linear dependence on magnetic field to high magnetic field. This is understandable in light of the fact that the terms quadratic in $B$ often cancel. For example, the energy difference between a pair of transitions from a common ground state to the two excited sublevels of a $\Gamma_{7}$ state will depend only on the term involving $g$ and not on the term involving $q$, which contributes to both sublevels equally.

In specifying transitions between states the quantum number $m\left(T_{d}\right)$ or the related quantum number $M\left(O_{h}\right)$ may be employed. The notation ${ }^{16}(i, f)$ where $i$ and $f$ are the subscripts of the irreducible representations $\left(O_{h}\right) \Gamma_{i}$ and $\Gamma_{f}$ of the initial and final states of an excitation experiment, will be used in this paper. In the notation of Schmitt et al. ${ }^{14}$ the order of the states is reversed. For example $(7,8)$ here is the transition denoted by $8 \rightarrow 7$ by Schmitt et al. ${ }^{14}$ The selection rules for $\boldsymbol{B} \|[001]$ allow transitions where $i=f$ for $\boldsymbol{E} \| \boldsymbol{B}$; for $\boldsymbol{E} \perp \boldsymbol{B}$, one of $i$ or $f$ is odd and the other is even.

The transitions allowed from the ground state to the lowest three states of odd parity are given in Fig. 2.

\section{EXPERIMENT}

The samples used in these experiments were grown by molecular-beam epitaxy on $450-\mu$ m-thick $\langle 100\rangle$ GaAs substrates with $\mathrm{Be}$ as the dopant. Details of the thickness of the doped layer and the Be concentrations are given in Table I for the two samples for which data are reported. The samples were wedged at $\simeq 2^{\circ}$ to suppress optical interference between the front and back faces. Detailed far-infrared absorption spectra for these samples have been published previously. $^{17}$

Measurements were made using two Fourier spectrometers equipped with globar light sources and liquid-helium cooled Si bolometer detectors. Appropriate beam splitters were employed to optimize the signal for the various spectral features examined.

For measurements at low magnetic fields $(<7 \mathrm{~T})$ a splitpair superconducting magnet was employed in the Voigt con-

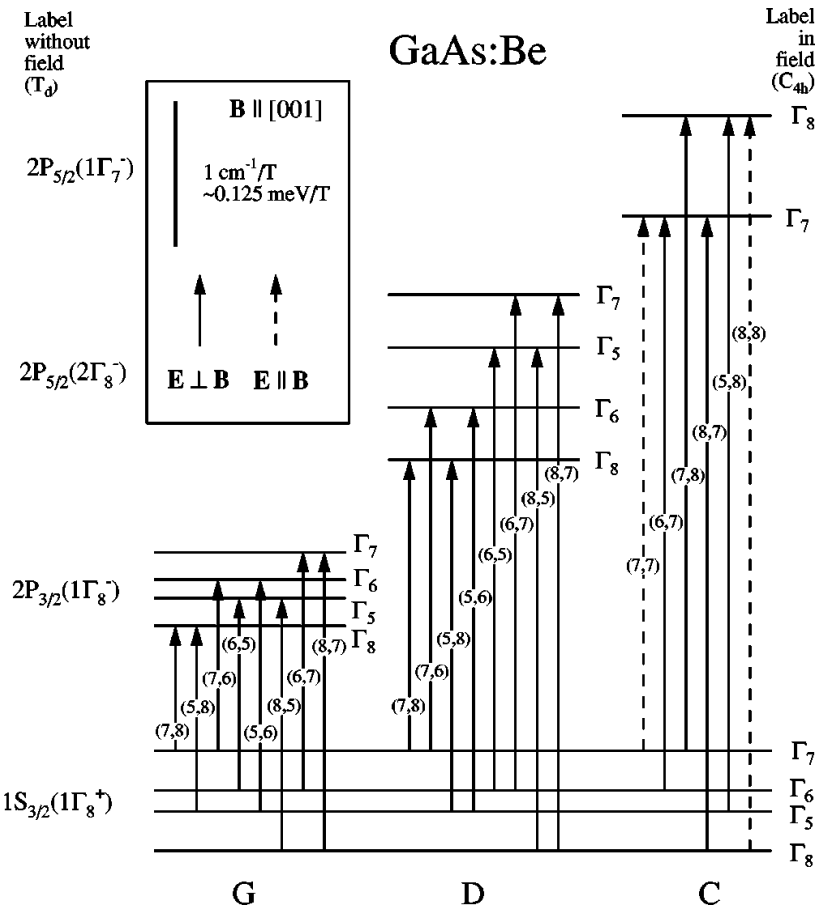

FIG. 2. Transitions for GaAs:Be in a magnetic field $\boldsymbol{B} \|[001]$. The allowed transitions are determined by group theory. All the transitions that might be observed in the geometries employed in the present experiments are shown. For the $G$ and the $D$ lines all measurements were for $\boldsymbol{E} \perp \boldsymbol{B}$. For the $C$ line data was obtained for both $\boldsymbol{E} \perp \boldsymbol{B}$ and $\boldsymbol{E} \| \boldsymbol{B}$. The ordering of the field-induced states and their splitting is as deduced from the experiments described in this paper. The energy splittings are shown to scale. The absolute values of the transition energies are not drawn to scale but can be obtained by combining the information here with that given in Fig. 1.

figuration which permitted data to be collected for both $\boldsymbol{E} \| \boldsymbol{B}$ and $\boldsymbol{E} \perp \boldsymbol{B}$. For measurements of Be impurity in GaAs at low magnetic fields it has been found that photothermal ionization spectroscopy (PTIS) is a more sensitive technique than absorption spectroscopy. ${ }^{18}$ It has also been found that the optimum impurity concentration is less for the PTIS measurements than for absorption measurements. Sample NU651 was used for the PTIS experiments at low magnetic field. The temperature is adjusted to optimize the PTIS experiment: at too low a temperature, thermal ionization is reduced; at too high a temperature, the lines broaden; higher temperatures yield relatively higher signals for deeper impurities. The ideal temperature for the PTIS measurements reported here was found to be in the range $16-18 \mathrm{~K}$.

Measurements at high magnetic field were made in both a 17.5 $\mathrm{T}$ superconducting magnet and a $30 \mathrm{~T}$ resistive magnet.

TABLE I. Samples employed in these experiments.

\begin{tabular}{lccc}
\hline \hline Sample & $\begin{array}{c}\text { Layer thickness } \\
(\mu \mathrm{m})\end{array}$ & $\begin{array}{c}\text { Be concentration } \\
\left(\text { atoms cm }^{-3}\right)\end{array}$ & $\begin{array}{c}\text { Be areal density } \\
\left(\text { atoms cm }^{-2}\right)\end{array}$ \\
\hline NU651 & 4 & $1.5 \times 10^{15}$ & $6.0 \times 10^{11}$ \\
NU652 & 3 & $2.3 \times 10^{16}$ & $6.9 \times 10^{12}$ \\
\hline \hline
\end{tabular}


The light was conducted to the sample at field center via a metal light pipe and a condenser cone. Measurements were made with the magnetic field parallel to the direction of light propagation (Faraday configuration) using nominally unpolarized radiation; in this geometry $\boldsymbol{E} \perp \boldsymbol{B}$.

The measured transmission $T$ of the sample was converted to the absorption coefficient $\alpha$ taking into account the finite thickness of the sample $t$ and the reflection at the sample surfaces, via the reflection coefficient $R$, according to the formula

$$
T=\frac{(1-R)^{2} \exp (\alpha t)}{\exp (2 \alpha t)-R^{2}} .
$$

The absorption coefficient calculated this way refers to the sample as a whole. Our main interest is in the absorption coefficient of the Be impurities confined to a layer of thickness $s$, rather than of the GaAs in this layer and in the substrate. Knowing the absorption coefficient $\alpha_{\mathrm{GaAs}}$ of GaAs from other measurements allows the extraction of the $a b-$ sorption coefficient of the Be impurity according to

$$
\alpha_{\mathrm{Be}}=\left(\alpha-\alpha_{\mathrm{GaAs}}\right) \frac{t}{s}
$$

\section{RESULTS AND DISCUSSION}

\section{A. Overview}

The Lyman series of Be impurity in GaAs occurs in the spectral range $120-240 \mathrm{~cm}^{-1}$. Transmission spectra of GaAs:Be in this range are presented in Fig. 3 from experiments in the high-field magnet for three representative magnetic fields: 0,18 , and $30 \mathrm{~T}$. All spectra were taken at a resolution of $1 \mathrm{~cm}^{-1}$, which is less than the spectral width of the lines. ${ }^{17}$

The unperturbed spectrum in Fig. 3 (0 T, full line) agrees well with spectra reported previously using other experimental arrangements. The strongest absorption feature is the $D$ line, at $166.8 \mathrm{~cm}^{-1}$. The second strongest feature is the $C$ line, at $182.3 \mathrm{~cm}^{-1}$. The $G$ line, whose position of $134.4 \mathrm{~cm}^{-1}$ is known from previous transmission and PTIS measurements, is difficult to resolve at $0 \mathrm{~T}$ in Fig. 3. The known energies of the $G, D$, and $C$ lines are indicated in the figure. The $G, D$, and $C$ lines are transitions from the ground state, $1 S_{3 / 2}\left(1 \Gamma_{8}^{+}\right)$, to the first three odd-parity excited states, $2 P_{3 / 2}\left(1 \Gamma_{8}^{-}\right), 2 P_{5 / 2}\left(2 \Gamma_{8}^{-}\right)$, and $2 P_{5 / 2}\left(1 \Gamma_{7}^{-}\right)$, respectively. The quality of the spectrum is high considering the limitations placed on the optical arrangements made necessary to locate the sample at the field center of the 30-T magnet. According to this experimental data the final states of the $G$ line and the $C$ line are separated from the final state of the $D$ line by 32.4 and $15.5 \mathrm{~cm}^{-1}$, respectively. These separations may be compared with the theoretical values of 33.7 and $15.1 \mathrm{~cm}^{-1}$ of Baldereschi and Lipari, ${ }^{12} 31.7$ and 12.0 of Schmitt et al., ${ }^{14}$ and 38.3 and 13.2 of Fiorentini. ${ }^{13}$

The second spectrum shown in Fig. 3 (18 T, dotted line) is at a field comparable to, but higher than, the highest field for which Zeeman data has been presented for the Be impurity

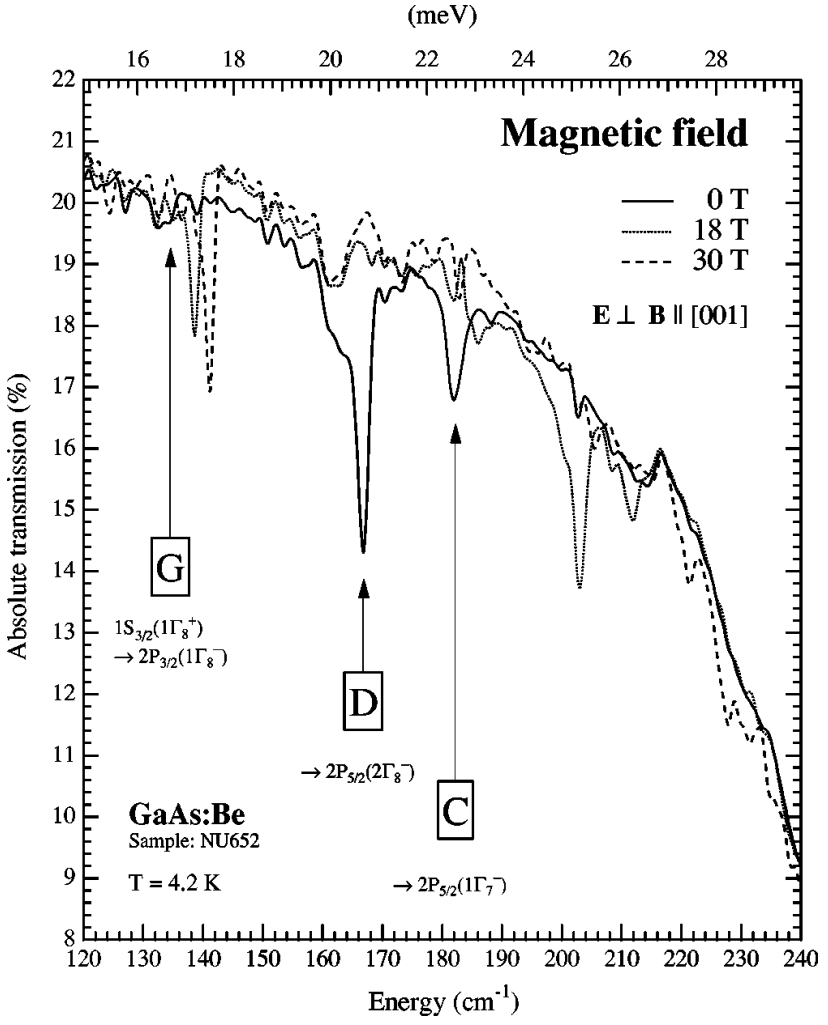

FIG. 3. Optical transmission spectra at low temperature of Be impurity in GaAs for three magnetic fields: 0,18 , and $30 \mathrm{~T}$. The $\mathrm{Be}$ concentration is $2.3 \times 10^{16} \mathrm{~cm}^{-3}$ in a $3-\mu \mathrm{m}$ doped layer, corresponding to an areal density of $6.9 \times 10^{12} \mathrm{~cm}^{-2}$; the layer is on a wedged substrate of nominally undoped GaAs of average thickness $364 \mu \mathrm{m}$.

in GaAs previously. A strong component of the $G$ line now appears at an energy $\sim 4 \mathrm{~cm}^{-1}$ greater than the energy of the unperturbed transition. In contrast, multiple components of the $D$ line, with individually much less absorption intensity than the unperturbed line, appear as a series of components spread over the range $154-186 \mathrm{~cm}^{-1}$. The $C$ line, like the $G$ line, exhibits one strong component; in contrast to the $G$ line, the strong component of the $C$ line moves at a much greater rate with field, and at $18 \mathrm{~T}$ is found $\sim 20 \mathrm{~cm}^{-1}$ above the unperturbed energy.

At the highest field for which data are given in Fig. 3 (30 T, dashed line) the $G$ line has strengthened further and shifted in a roughly linear fashion, now being $\sim 7 \mathrm{~cm}^{-1}$ from its unperturbed position. The $G$ line absorption intensity increases with field. The $D$ components continue to spread and the strong $C$ component continues to higher field.

While the chief spectral range of interest is $120-240 \mathrm{~cm}^{-1}$, the experimental arrangement permitted measurements to be made over the range $50-900 \mathrm{~cm}^{-1}$, except for two windows for which the transmission was zero in the region of GaAs reststrahlen, $\simeq 260-300 \mathrm{~cm}^{-1}$, and in the region of the $3.5-\mu \mathrm{m}$-mylar beam splitter minimum, $\simeq 700-720 \mathrm{~cm}^{-1}$. Over this wide energy range, no additional features associated with the Be impurity were observed, nor any that moved with the magnetic field, except 


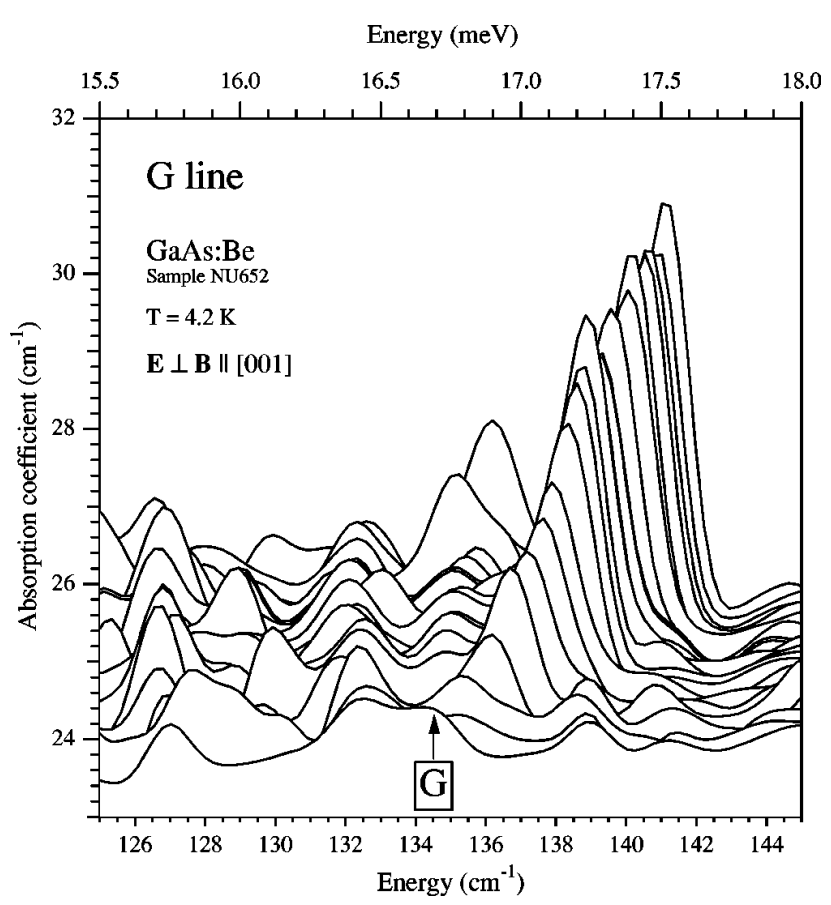

FIG. 4. Development with the magnetic field of the $G$ line $\left[1 S_{3 / 2}\left(1 \Gamma_{8}^{+}\right) \rightarrow 2 P_{3 / 2}\left(1 \Gamma_{8}^{-}\right)\right.$transition $]$of the Be impurity in GaAs. From front to back the spectra are, respectively, for $0,2,4,6,8,10$, $12,14,16,18,19,20,21,22,23,24,25,26,27,28,29$, and $30 \mathrm{~T}$. The ordinate applies to the unperturbed spectrum. Each spectrum is offset by an amount proportional to the magnetic field at which it was taken. The unperturbed energy of the $G$ line is also indicated.

for one high-field feature that will be discussed below. In this respect, the behavior of GaAs:Be is very different to, for example, InP:Zn, which was measured in the same apparatus at about the same time. ${ }^{19}$

\section{B. $G$ line}

The development of the $G$ line with the magnetic field is shown in Fig. 4. The most striking feature is an absorption peak that moves to higher energy and increases in strength as the magnetic field increases. Other absorption features that move systematically with field may be identified but a relatively large noise-to-signal in this part of the spectrum limits the number of components that may be unambiguously identified.

The variation of the position of the strongest absorption peak with the magnetic field is given in Fig. 5. Also shown is previous data to $17.5 \mathrm{~T}^{10}$ The previous data is rather linear but with a slight negative curvature; that is, the rate of increase in energy with the magnetic field decreases slightly as the field increases. The present data reproduce this trend to $17.5 \mathrm{~T}$ and beyond, until about $20 \mathrm{~T}$. Unexpectedly, beyond $20 \mathrm{~T}$, the trajectory of the data straightens out, and these data, especially at the highest fields, are well fitted by a straight line which passes through the unperturbed energy of the $G$ line at $B=0$.

On the basis of comparison with the previous work the strong feature was previously identified ${ }^{10}$ as the $(8,5)$ transi-

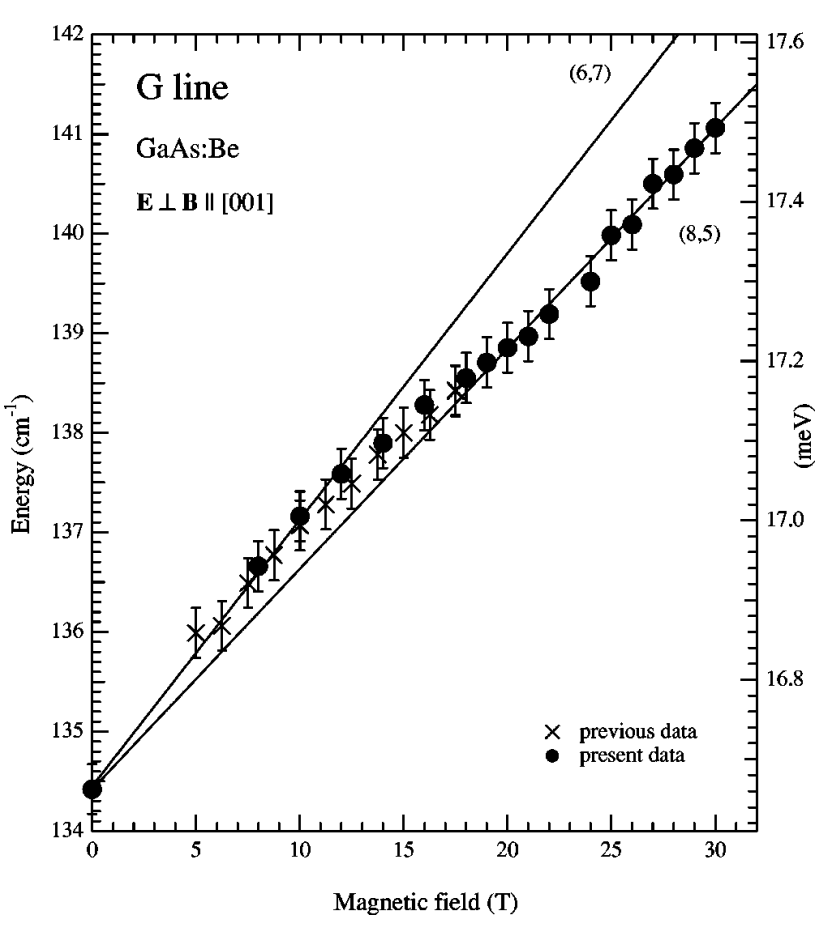

FIG. 5. Energy of the strongest component of the $G$ line as a function of magnetic field. The crosses are data obtained previously (Ref. 10) up to $17.5 \mathrm{~T}$ using a superconducting magnet. The circles are present data collected in a resistive magnet to $30 \mathrm{~T}$.

tion, or $X$ in the notation of Atzmüller $e t a l^{7}$ for the $G$ line of GaAs:C. In the light of the new data beyond $20 \mathrm{~T}$ this assignment warrants reappraisal. The linear behavior of the high-field data, extrapolating back to the zero-field $G$ line energy, suggests the origin of that data as one of the fieldinduced components of the $G$ line. That the high-field transition increases in strength with field suggests its initial state is the lowest sublevel of the field-split ground state. This is consistent with the high-field data being the $(8,5)$ transition. The higher-energy component, which dominates at lower fields, but then loses intensity from about $10 \mathrm{~T}$ and becomes undetectable at $20 \mathrm{~T}$, is attributed to the $(6,7)$ transition, or $X^{\prime}$ in the notation of Atzmüller et al. ${ }^{7}$ The region of negative curvature, which might previously have been attributed to a gradual introduction of quadratic or higher terms in energies of the sublevels of the ground or excited states as a function of magnetic field, is now regarded as the region of change from one transition to a second, both of which are linear in field. The data for $(8,5)$ and $(6,7)$ are fitted by straight lines with slopes $(0.268 \pm 0.009) \mathrm{cm}^{-1} / \mathrm{T}$ and $(0.221$ $\pm 0.020) \mathrm{cm}^{-1} / \mathrm{T}$, respectively. These transitions of themselves are of limited usefulness in determining $g$ factors, since they have no initial or final states in common. However, they may be combined with other information to obtain $g$ factors, as will be discussed in the final section of the paper.

\section{C. $D$ line}

The development of the $D$ line with the magnetic field is shown in Fig. 6. As with the $G$ line, the strongest component 


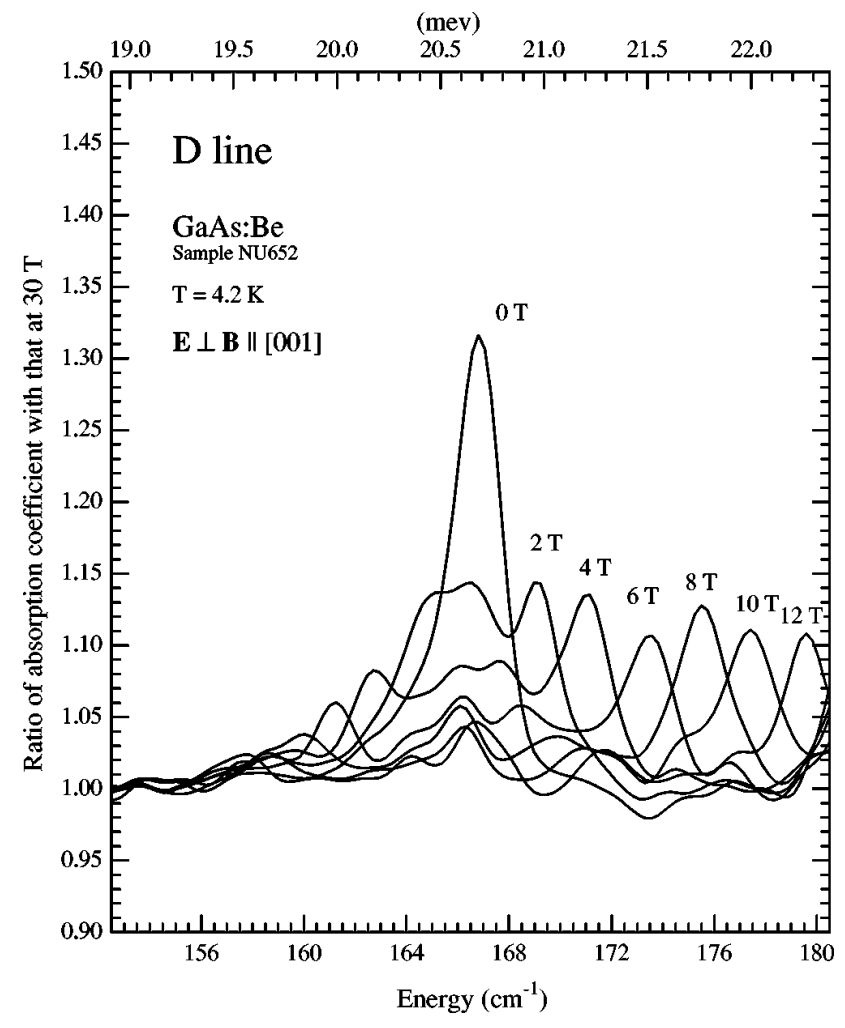

FIG. 6. Development with the magnetic field of the $D$ line $\left[1 S_{3 / 2}\left(1 \Gamma_{8}^{+}\right) \rightarrow 2 P_{3 / 2}\left(2 \Gamma_{8}^{-}\right)\right.$transition] of the Be impurity in GaAs.

proceeds to higher field. More components are observed for the $D$ line than are observed for the $G$ line - up to the eight permitted by the selection rules. The behavior of the components of the $D$ line to $17.5 \mathrm{~T}$ has been reported in detail before $^{10}$ and the previous data is reproduced in Fig. 7 along with the new data. It will be observed that the two datasets agree well. At high field, the main components are associated with the transitions identified earlier as $(8,7),(6,7)$, and $(5,6)$. As with the $G$ line, there are no initial or final states in common in the observed transitions, meaning the splitting of particular ground or excited-state sublevels cannot be determined by this information alone. This point is discussed further in the final section of the paper.

\section{D. $C$ line}

In interpreting the data presented thus far one is faced with two serious shortcomings. First, the geometry of the experimental apparatus used in the high-field magnet does not permit measurements for which $\boldsymbol{E} \| \boldsymbol{B}$. As a consequence, no transitions are observed between a common ground(excited-) state sublevel and a pair of excited- (ground-) state sublevels that differ only in the sign of the quantum number $m$ or $M$. Such a pair allows the determination of the energy splitting of the sublevels of the excited- (ground-) state involved. Secondly, of the eight lines expected for $\boldsymbol{E} \perp \boldsymbol{B}$, far fewer are observed at high magnetic field.

These deficiencies are largely overcome by an examination of the $C$ line at low magnetic field $(<7 \mathrm{~T})$ in the splitpair superconducting magnet which permitted data to be col-

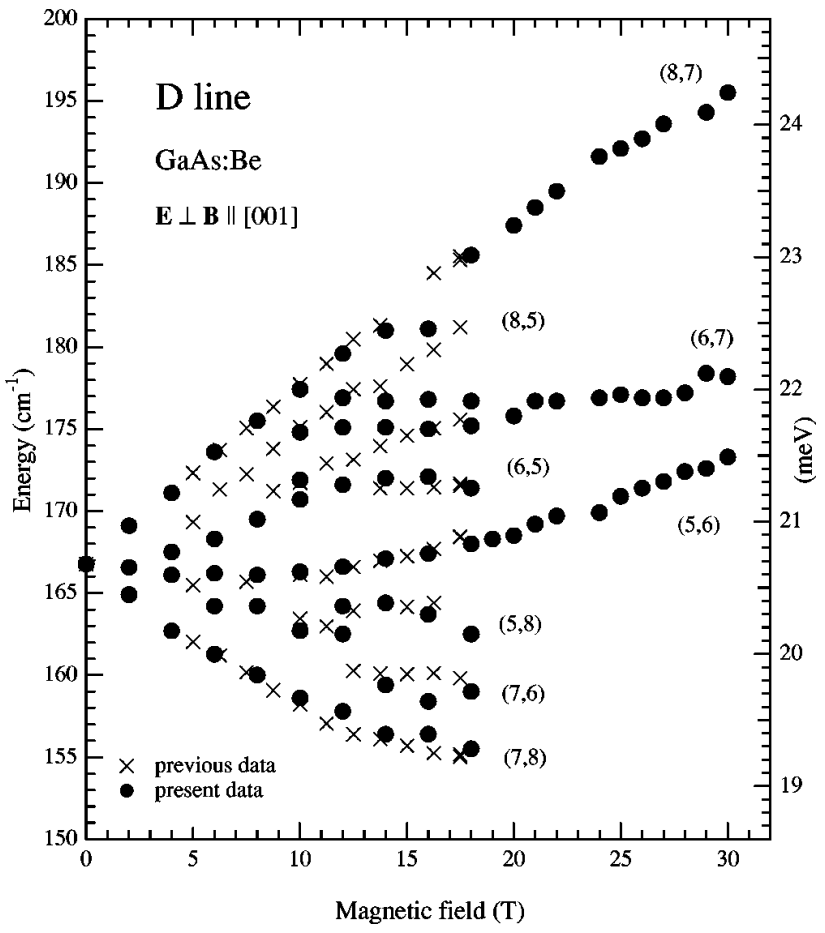

FIG. 7. Energy of the components of the $D$ line as a function of magnetic field. The crosses are data obtained previously (Ref. 10) up to $17.5 \mathrm{~T}$ using a superconducting magnet. The circles are present data collected in a resistive magnet to $30 \mathrm{~T}$.

lected for both $\boldsymbol{E} \| \boldsymbol{B}$ and $\boldsymbol{E} \perp \boldsymbol{B}$. In contrast to the cases of the $G$ line and the $D$ line, which have $\Gamma_{8}$ final states, the final state of the $C$ line is $\Gamma_{7}$, which is doubly, rather than fourfold, degenerate, and so splits under magnetic field into only two sublevels, rather than four. The selection rules in this case permit two transitions for $\boldsymbol{E} \| \boldsymbol{B}$ and four for $\boldsymbol{E} \perp \boldsymbol{B}$. As will be demonstrated, all but one of these transitions are resolved, and the disposition of the observed transitions allows a reliable determination of the splitting of both the ground state $1 S_{3 / 2}\left(1 \Gamma_{8}^{+}\right)$and the excited state $2 P_{5 / 2}\left(1 \Gamma_{7}^{-}\right)$.

The $C$ line is especially fruitful for examination for acceptors in GaAs in view of its relative isolation. According to the calculation of Fiorentini, ${ }^{13}$ the $2 P_{5 / 2}\left(1 \Gamma_{7}^{-}\right)$state is separated in energy by $0.44 \mathrm{meV}$ from the next-nearest state, namely, $3 P_{3 / 2}\left(3 \Gamma_{8}^{-}\right)$. In contrast, for acceptors in $\mathrm{Ge}$ the separation in energy of these states is an order of magnitude less. As an example of the complications that may be involved, we cite the case of $\mathrm{Zn}^{-}$acceptor in $\mathrm{Ge}$, whose $C$ manifold has been deduced from piezospectroscopy ${ }^{20}$ to comprise at least components $1 \Gamma_{7}^{-}, 3 \Gamma_{8}^{-}, 3 \Gamma_{8}^{+}$, and $4 \Gamma_{8}^{+}$.

Figure 8 shows the PTIS spectra in the region of the $C$ line for $B=3 \mathrm{~T}, \boldsymbol{B} \|[001]$ for both $\boldsymbol{E} \| \boldsymbol{B}$ and $\boldsymbol{E} \perp \boldsymbol{B}$. For all fields $1 \mathrm{~T} \leqslant B \leqslant 6 \mathrm{~T}$ the $\boldsymbol{E} \| \boldsymbol{B}$ spectra exhibit two components, as expected on the basis of the selection rules. At $B$ $=1 \mathrm{~T}$ the $\boldsymbol{E} \perp \boldsymbol{B}$ components are not resolved, but for fields $2 \mathrm{~T} \leqslant B \leqslant 6 \mathrm{~T}$ three components are observed, whereas the selection rules permit four.

The positions of the PTIS peaks for the components of the $C$ line are plotted against magnetic field in Fig. 9. The data 


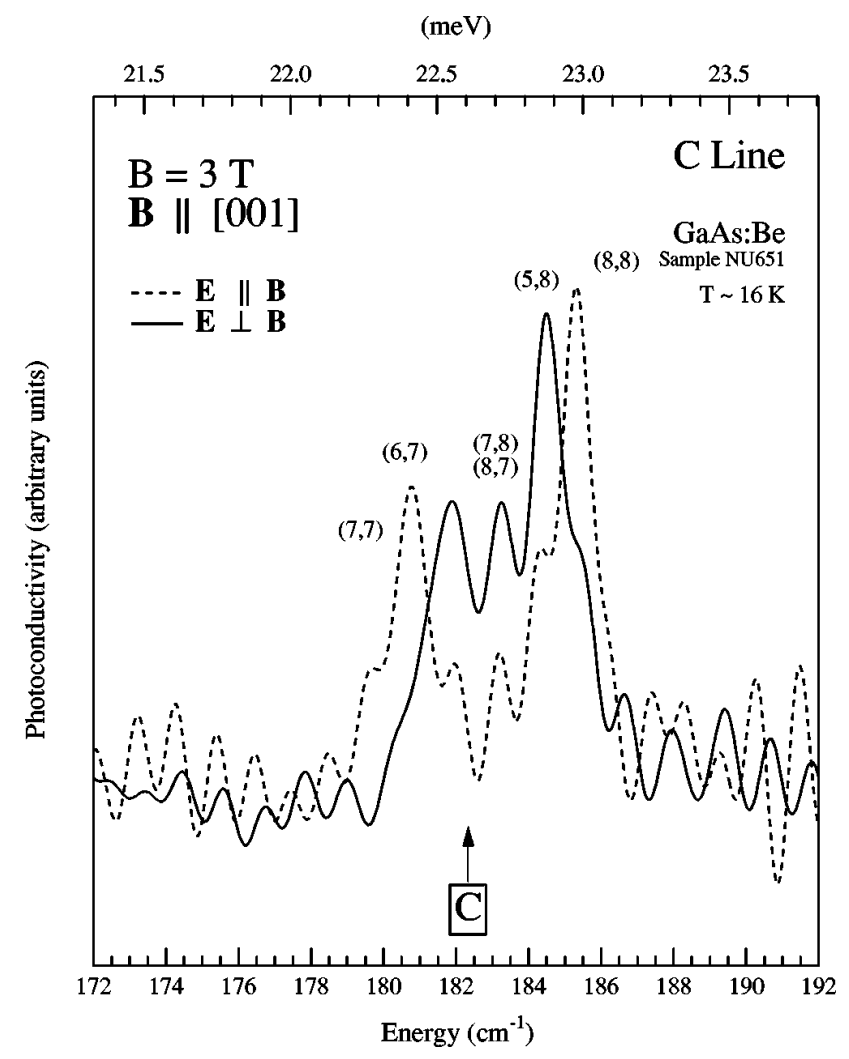

FIG. 8. The PTIS spectra observed in a magnetic field of $B$ $=3 T$ for $\boldsymbol{B} \|[001]$ in the region of the $C$ line. The position of the unperturbed $C$ line is indicated.

are well described by second-order polynomials with $B$, as indicated by the full lines in the figure. The two transitions expected on the basis of the selection rules for $\boldsymbol{E} \| \boldsymbol{B}$ turn out to be the highest- and the lowest-energy transitions. Significantly, only three of the four transitions expected for $\boldsymbol{E} \perp \boldsymbol{B}$ are observed. In attempting to locate the missing transition, it is noted that, within the linear approximation, the average energy of sublevels which differ only in quantum number $m$ will be the same. As an extension of this principle, the average energy of the $\boldsymbol{E} \| \boldsymbol{B}$ transitions should coincide with the average energy of the $\boldsymbol{E} \perp \boldsymbol{B}$ transitions at any given field. The average energy of the two $\boldsymbol{E} \| \boldsymbol{B}$ transitions is indicated by the dashed line in Fig. 9. This average energy is seen to coincide very closely with the intermediate of the three transitions observed for $\boldsymbol{E} \perp \boldsymbol{B}$. This recognition immediately suggests that the observed intermediate energy transition for $\boldsymbol{E} \perp \boldsymbol{B}$ is the superposition of two unresolved transitions. The symmetric disposition of the higher- and lower-energy transitions for $\boldsymbol{E} \perp \boldsymbol{B}$ is also consistent with the "missing" fourth transition for $\boldsymbol{E} \perp \boldsymbol{B}$ coinciding with the intermediate-energy transition observed. Two $\boldsymbol{E} \perp \boldsymbol{B}$ transitions will coincide in the way proposed if two of the sublevels of the ground state separate with the magnetic field at the same rate as do the two sublevels of the excited state. We note in the calculations of Schmitt et al. ${ }^{14}$ that the $\Gamma_{8}$ and $\Gamma_{7}$ components of the fourfold degenerate ground state $1 S_{3 / 2}\left(1 \Gamma_{8}^{+}\right)$are predicted to split at a rate similar to the $\Gamma_{7}$ and $\Gamma_{8}$ components of the excited state $2 P_{5 / 2}\left(1 \Gamma_{7}^{-}\right)$.

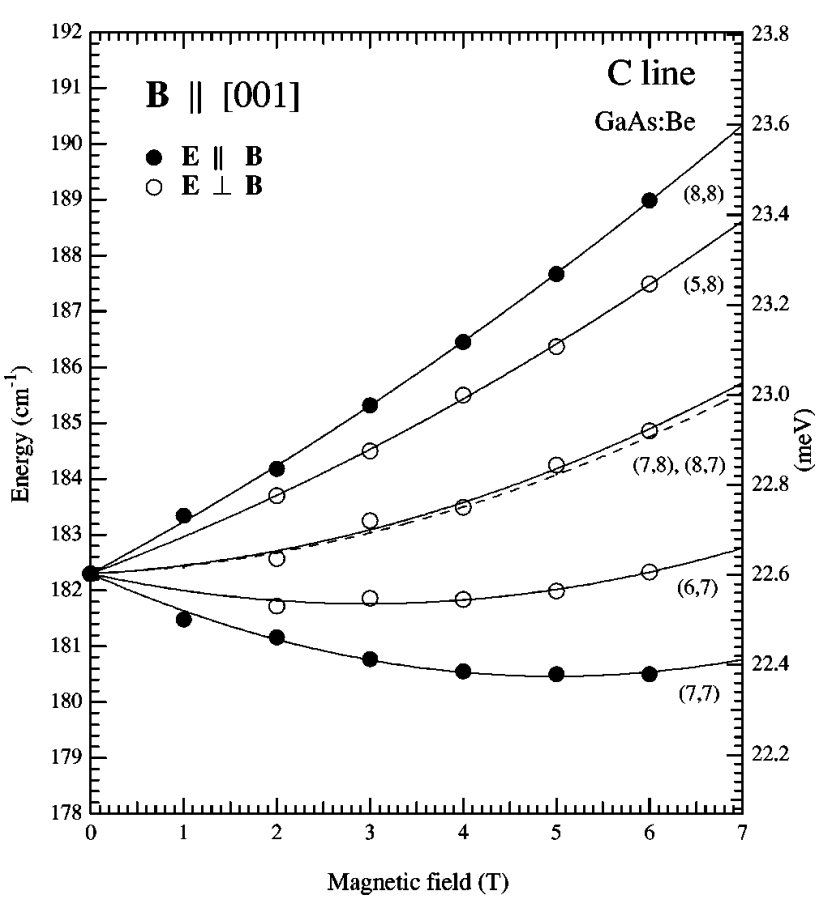

FIG. 9. Energy dependence of the components of the $C$ line on the magnetic field. The full lines indicate the second-order polynomial fits to the data. The dashed line represents a fit to the average energy of the highest- and lowest-energy components.

Given this interpretation, we may now obtain the splittings of all the components of the ground state and the excited state. In other words, it is possible to determine the parameters $g_{1}^{\prime}$ and $g_{2}^{\prime}$ for the $1 S_{3 / 2}\left(1 \Gamma_{8}^{+}\right)$state and $g^{C}$ for the $2 P_{5 / 2}\left(1 \Gamma_{7}^{-}\right)$state. We take the order of the ground-state sublevels to be as given by Schmitt et al., ${ }^{14}$ that is, $\Gamma_{7}, \Gamma_{6}, \Gamma_{5}$, and $\Gamma_{8}$. The splitting of the two outer sublevels of the ground state, which is the same as the splitting of the two sublevels of the excited state within the experimental error, and the splitting of the two inner sublevels of the ground state, are determined from the data in Fig. 9 and are given in Fig. 10. The states and transitions have been labeled in Figs. $8-10$ on the basis of the transition scheme discussed above. It is emphasized that, in analyzing the experimental data, the only data required from without is the ordering of the sublevels of the ground state, which was first determined by Bimberg $^{21}$ using electron-acceptor photoluminescence. Given this, the present experimental data permits the determination of the order of the sublevels in the excited state and the linear energy splitting of all the sublevels in the two manifolds.

Absorption measurements taken at high magnetic fields in the region of the $C$ line are presented in Fig. 11. The most prominent transition is the line identified as the $(5,8)$ transition. This identification is on the basis of the low-field PTIS data, also shown in the figure. The second most prominent transition is identified as the coincident lines $(8,7)$ and $(7,8)$. Even at high field these two transitions are not separated.

Another distinctive line appears at high energy. The slope of the line position plotted against magnetic field, and the identification of the transitions made above, mean it is un- 


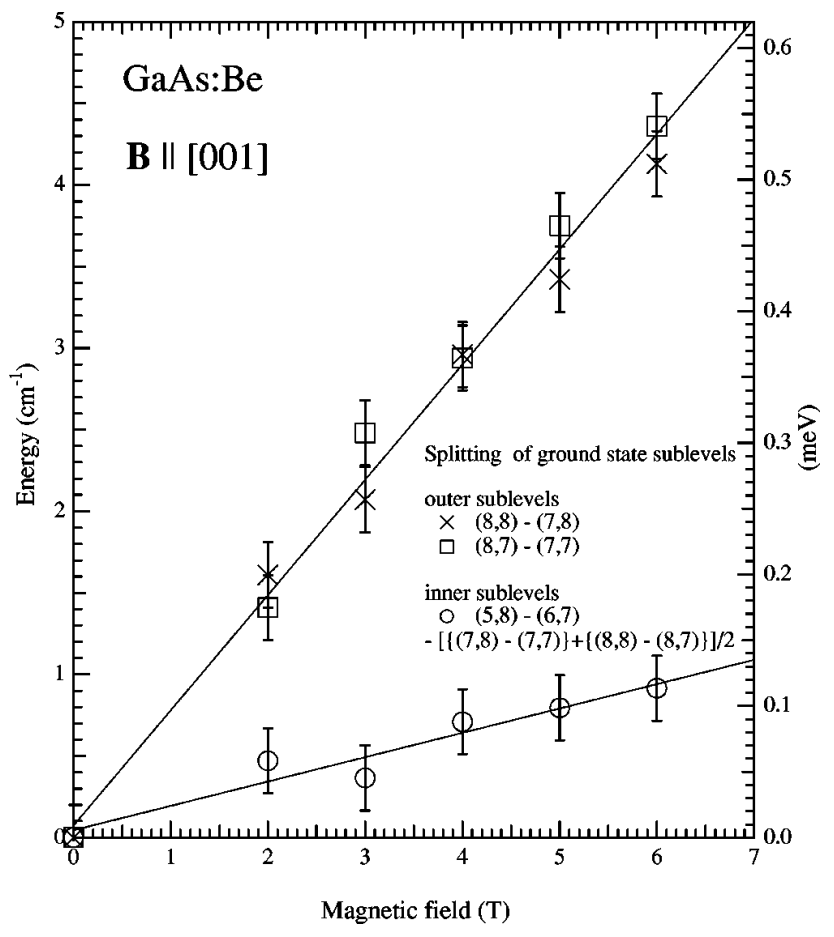

FIG. 10. Energy splitting of the sublevels of the ground state as deduced from the data in the previous figure. The full lines shown are linear fits to the data.

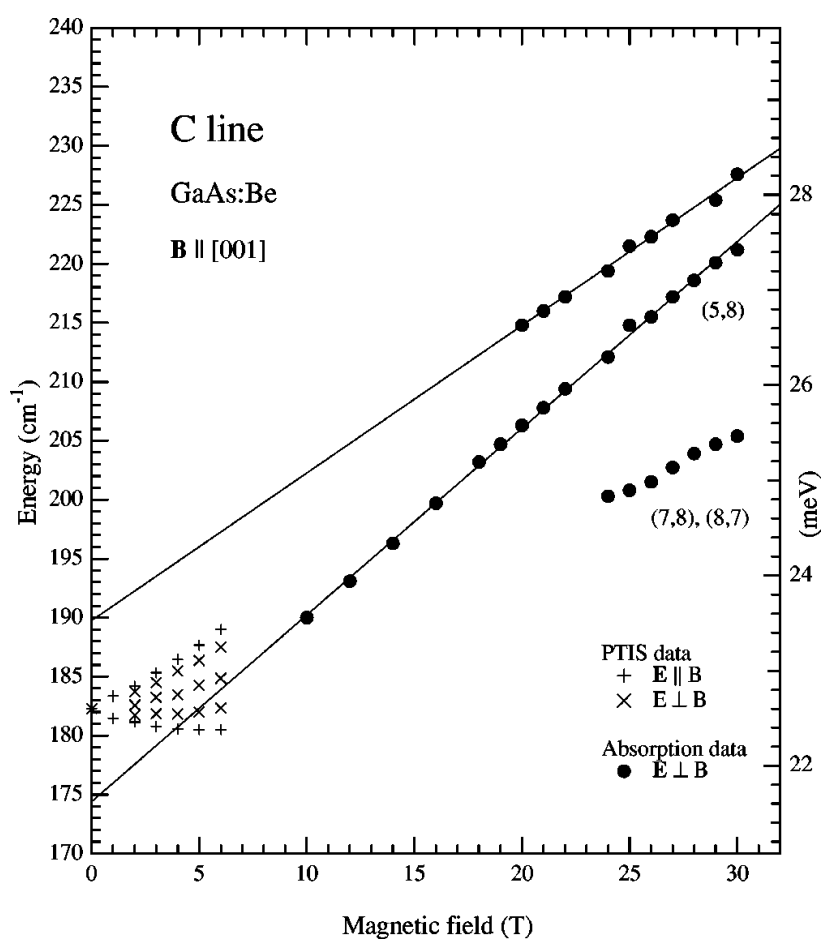

FIG. 11. Energy of the components of the $C$ line as a function of the magnetic field. The crosses are the PTIS data shown in Fig. 9. The circles are present data collected in a resistive magnet to $30 \mathrm{~T}$.

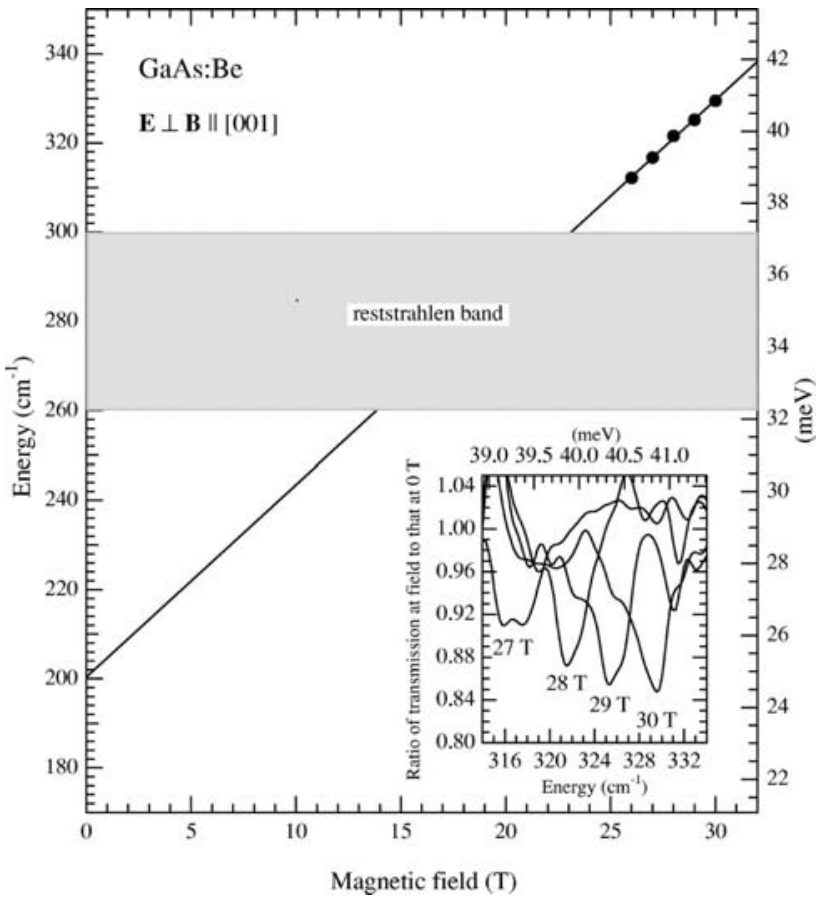

FIG. 12. Transmission spectra of the high-energy feature observed at high magnetic field. A linear extrapolation to zero field and the reststrahlen region are also indicated.

likely to arise from the $C$ line. It is presumed to arise from the next state, $3 P_{3 / 2}\left(3 \Gamma_{8}^{-}\right)$. A linear interpolation of this line to zero field gives an intercept of $189.75 \mathrm{~cm}^{-1}$, while that of the line identified as $(5,8)$ gives $174.41 \mathrm{~cm}^{-1}$. This difference in intercepts of $15.34 \mathrm{~cm}^{-1}=1.9 \mathrm{meV}$ and is rather large compared to the separation between the states of 0.44 meV given by Fiorentini; ${ }^{13}$ the high-energy line may thus have another origin to that proposed here.

\section{E. High-field line}

An unexpected feature appears at the highest field $(30 \mathrm{~T})$ above the reststrahlen band, as shown in Fig. 12. It decreases in energy with decreasing field, at a rate of (4.31 $\pm 0.12) \mathrm{cm}^{-1} / \mathrm{T}$ rapidly moving into the reststrahlen band where it is unobservable. The feature does not appear to emerge from below the reststrahlen band as the field is reduced to zero. It would be of great interest to follow this feature to higher fields although to do so would involve considerable technical difficulty. A linear fit of the energy with field extrapolates to a zero-field energy of $205 \mathrm{~cm}^{-1}$ $=25.4 \mathrm{meV}$. It is speculated that this feature is related to the first Landau level of the valence band and tentatively suggested that its zero-field position gives a measure of the ground state of the Be acceptor and may be compared with the values given in Fig. 1.

\section{F. $g$ factors}

The outer ground-state energy splitting, $E\left(\Gamma_{7}\right)-E\left(\Gamma_{8}\right)$, is determined by the linear fit to the data given in Fig. 10 to be $(0.707 \pm 0.015) \mathrm{cm}^{-1} / \mathrm{T}$. Thus, 
TABLE II. $g$ factors for some acceptor states in GaAs.

\begin{tabular}{llccc}
\hline \hline & & $\begin{array}{c}\text { Theory } \\
\text { Generic } \\
\text { acceptor }\end{array}$ & $\begin{array}{c}\text { Experiment } \\
\text { GaAs:C }\end{array}$ & $\begin{array}{c}\text { Experiment } \\
\text { (this work) } \\
\text { GaAs:Be }\end{array}$ \\
\hline $1 \Gamma_{8}^{+}$ & $g_{1}^{\prime}$ & +0.281 & $+0.30 \pm 0.08$ & $+0.30 \pm 0.05$ \\
& $g_{2}^{\prime}$ & +0.1147 & $+0.09 \pm 0.05$ & $+0.09 \pm 0.03$ \\
$1 \Gamma_{8}^{-}$ & $g_{1}^{G}$ & -2.402 & $-2.38 \pm 0.03$ & $-1.63 \pm 0.10$ \\
& $g_{2}^{G}$ & +1.024 & $+1.01 \pm 0.01$ & $+0.64 \pm 0.06$ \\
$2 \Gamma_{8}^{-}$ & $g_{1}^{D}$ & -3.6420 & $-3.15 \pm 0.15$ & $-2.88 \pm 0.19$ \\
& $g_{2}^{D}$ & +1.8259 & $+1.57 \pm 0.06$ & $+1.47 \pm 0.10$ \\
$1 \Gamma_{7}^{-}$ & $g^{C}$ & & & $+1.51 \pm 0.03$ \\
\hline \hline
\end{tabular}

Ref. 7.

${ }^{\mathrm{b}}$ Ref. 14

$$
3 g_{1}^{\prime}+\frac{27}{4} g_{2}^{\prime}=1.514 \pm 0.032
$$

Likewise, the inner ground-state energy splitting, $E\left(\Gamma_{6}\right)$ $-E\left(\Gamma_{5}\right)$, increases at a rate of $(0.150 \pm 0.021) \mathrm{cm}^{-1} / \mathrm{T}$, giving

$$
g_{1}^{\prime}+\frac{1}{4} g_{2}^{\prime}=0.320 \pm 0.044
$$

From these two relations the $g$ factors of the ground state may be deduced. The values are given in Table II and are found to be identical to those determined by Atzmüller et al. ${ }^{7}$ for carbon impurity in GaAs.

Within the experimental error the splitting of the $2 P_{5 / 2}\left(1 \Gamma_{7}^{-}\right)$state is determined to be the same as that of the outer ground state. It follows directly that

$$
g^{C}=1.514 \pm 0.032 \text {. }
$$

The data presented for the $G$ line and the $D$ line may now also be analyzed.

All the components of the $D$ line are observed and so the possibility exists to group these in pairs to minimize effects quadratic in $B$. The energy differences $(8,7)-(7,8)$ and $(6,5)-(5,6)$ were chosen for analysis. The former represents the sum of the splitting of the outer sublevels of the excited state and the splitting of the outer sublevels of the ground state; the latter gives the difference between the splitting of the inner sublevels of the excited state and the splitting of the inner sublevels of the ground state. When plotted against field these energy differences begin to diverge from a linear dependence on $B$ at around $10 \mathrm{~T}$. The slopes of the linear fits in the range $0-10 \mathrm{~T}$ are $(1.88 \pm 0.06) \mathrm{cm}^{-1} / \mathrm{T}$ and $(0.44$ $\pm 0.04) \mathrm{cm}^{-1} / \mathrm{T}$, respectively. From these slopes and Eqs. (8) and (9), the $g$ factors of the excited state of the $D$ line may be determined. The results are given in Table II. The results are in excellent agreement with the previous determinations made using transmission and photoconductivity measurements in a superconducting solenoid to $6 \mathrm{~T}$ which yielded $g_{1}^{D}=-2.81$ and $g_{2}^{D}=+1.40 .{ }^{22}$ The values are somewhat smaller in magnitude than, but agree, within error, with the experimental results of Atzmüller et al. ${ }^{7}$ for GaAs:C,

which in turn are smaller in magnitude than the theoretical values calculated by Schmitt et al. ${ }^{14}$ for a generic acceptor in GaAs.

The $g$ factors deduced above for the ground state $1 S_{3 / 2}\left(1 \Gamma_{8}^{+}\right)$and for the second excited state $2 P_{5 / 2}\left(2 \Gamma_{8}^{-}\right)$of $\mathrm{GaAs}: \mathrm{Be}$ agree well with those of GaAs:C. In contrast, the splitting of the first excited state $2 P_{3 / 2}\left(1 \Gamma_{8}^{-}\right)$appears to be rather different in the two systems. Atzmüller et al. ${ }^{7}$ observe all four expected transitions with positive energy dependencies with field and denote these, in order of increasing energy, $W, X, X^{\prime}$, and $Y$. In the present notation these transitions correspond to $(5,6),(8,5),(6,7)$, and $(8,7)$, respectively. The slopes for the energy dependence with field for these transitions $^{7}$ are, respectively, 0.084, 0.320, 0.397, and $0.848 \mathrm{~cm}^{-1} / \mathrm{T}$. The two lines shown in the present Fig. 5 have slopes of $(0.268 \pm 0.009) \mathrm{cm}^{-1} / \mathrm{T}$ and $(0.221$ $\pm 0.020) \mathrm{cm}^{-1} / \mathrm{T}$. These data are not in agreement with any of the slopes reported for GaAs:C. Given that the groundstate $g$ factors are identical for GaAs:Be and GaAs:C (see Table II), one concludes that the splitting of the first excited state must be different in the two impurity systems. It should be noted that the discrepancy is evident in the raw experimental data and does not depend on the interpretation of which transitions give rise to which spectral lines or on the fact that in the present experiments fewer spectral lines are observed than in the former.

From the depopulation of the upper sublevels of the ground state with the magnetic field, it is deduced that the prominent component of the $G$ line at high field must be a transition from the lowest sublevel. There are only two possibilities for the transition, $(8,7)$ or $(8,5)$. In both the experimental work of Atzmüller et al. ${ }^{7}$ for GaAs:C and the theory of Schmitt et al. ${ }^{14}$ for the generic acceptor in GaAs, the $(8,7)$ transition is the highest-energy transition, which is incompatible with the present data, where the low-field transition has a higher energy than the high-field transition under discussion. Moreover, the slope of this transition, $(0.221$ $\pm 0.020) \mathrm{cm}^{-1} / \mathrm{T}$, differs greatly from the slope of the $(8,7)$ transition in GaAs:C, of $0.848 \mathrm{~cm}^{-1} / \mathrm{T}$. On these grounds it is concluded that the high-field transition cannot be $(8,7)$, but is $(8,5)$. Using similar reasoning it is concluded that the lowfield transition is $(6,7)$. From the slopes determined from Fig. 5 and the data in Eqs. (8) and (9), $g_{1}^{G}$ and $g_{2}^{G}$ are calculated; the results are given in Table II. 


\section{CONCLUSION}

Employing the $C$ line, or $1 S_{3 / 2}\left(1 \Gamma_{8}^{+}\right) \rightarrow 2 P_{5 / 2}\left(1 \Gamma_{7}^{-}\right)$transition, the ground-state $g$ factors of $\mathrm{Be}$ in $\mathrm{GaAs}$ have been determined. Somewhat surprisingly, identical results are obtained to those reported by Atzmüller et al. ${ }^{7}$ obtained from studying different transitions (the $D$ and $G$ lines) of a different impurity (carbon) in GaAs. The $g$ factor of the excited state of the $C$ line has been determined. The $g$ factors of the $2 P_{5 / 2}\left(2 \Gamma_{8}^{-}\right)$state, corresponding to the $D$ line, have been determined in the linear region of $0-10 \mathrm{~T}$ and are in excellent agreement with values determined earlier ${ }^{22}$ to $6 \mathrm{~T}$ using transmission and PTIS and agree well with the values reported for carbon impurity. ${ }^{7}$ The field splitting of the components of the $G$ line is different to that reported for GaAs:C. The low-field transition has been reassigned and the $g$ factors for the $2 P_{3 / 2}\left(1 \Gamma_{8}^{-}\right)$state have been redetermined from the experimental data. An unexpected transition which appears only at high field ( $>26 \mathrm{~T})$ is attributed to valence-band Landau states.

\section{ACKNOWLEDGMENTS}

This work was supported by the Australian Research Council, the University of Wollongong, and the EPSRC (UK). A part of the work was performed at the National High Magnetic Field Laboratory, which is supported by NSF Cooperative Agreement No. DMR-9527035 and by the State of Florida. B. Oborn assisted with some preliminary data analysis. We are grateful to P. E. Simmonds and P. Fisher for many valuable comments.
*Electronic address: roger@uow.edu.au

${ }^{1}$ P. Fisher and A. K. Ramdas, in Physics of the Solid State, edited by S. Balakrishna, M. Krishnamurthi, and B. Ramachandra Rao (Academic, New York, 1969), pp. 149-182.

${ }^{2}$ A. K. Ramdas and S. Rodriguez, Rep. Prog. Phys. 44, 1297 (1981).

3 The 9th International Conference on Shallow-Level Centers in Semiconductors, edited by M. Suezawa, H. Nakata, and H. Katayama-Yoshida (Elsevier Science, Oxford, 2001), and references therein to previous conferences in the series.

${ }^{4}$ G. E. Stillman, D. M. Larsen, C. M. Wolfe, and R. C. Brandt, Solid State Commun. 9, 2245 (1971).

${ }^{5}$ H. Sigg, J. A. A. J. Perenboom, P. Pfeffer, and W. Zawadzki, Solid State Commun. 61, 685 (1987).

${ }^{6}$ R. J. Heron, R. A. Lewis, P. E. Simmonds, R. P. Starrett, A. V. Skougarevsky, R. G. Clark, and C. R. Stanley, J. Appl. Phys. 85, 893 (1999).

${ }^{7}$ R. Atzmüller, M. Dahl, J. Kraus, G. Schaak, and J. Schubert, J. Phys.: Condens. Matter 3, 6775 (1991).

${ }^{8}$ R. F. Kirkman, R. A. Stradling, and P. J. Lin-Chung, J. Phys. C 11, 419 (1978).

${ }^{9}$ J. Schubert, M. Dahl, and E. Bangert, in High Magnetic Fields in Semiconductor Physics II, edited by G. Landwehr (Springer-
Verlag, Berlin, 1989), pp. 567-572.

${ }^{10}$ R. A. Lewis and M. Henini, Solid State Commun. 112, 25 (1999).

${ }^{11}$ A. Baldereschi and N. O. Lipari, Phys. Rev. B 8, 2697 (1973).

${ }^{12}$ A. Baldereschi and N. O. Lipari, Phys. Rev. B 9, 1525 (1974).

${ }^{13}$ V. Fiorentini, Phys. Rev. B 51, 10161 (1995).

${ }^{14}$ W. O. G. Schmitt, E. Bangert, and G. Landwehr, J. Phys.: Condens. Matter 3, 6789 (1991).

${ }^{15}$ A. K. Bhattacharjee and S. Rodriguez, Phys. Rev. B 6, 3836 (1972).

${ }^{16}$ R. J. Baker, P. Fisher, C. A. Freeth, D. S. Ryan, and R. E. M. Vickers, Solid State Commun. 93, 353 (1995).

${ }^{17}$ R. A. Lewis, T. S. Cheng, M. Henini, and J. M. Chamberlain, Phys. Rev. B 53, 12829 (1996).

${ }^{18}$ R. A. Lewis, P. E. Simmonds, T. M. Silver, T. S. Cheng, M. Henini, and J. M. Chamberlain, in 7th International Conference on Shallow-Level Centers in Semiconductors, edited by C. A. J. Ammerlaan and B. Pajot (World Scientific, Singapore, 1997), pp. 257-262.

${ }^{19}$ R. A. Lewis (unpublished).

${ }^{20}$ G. Piao, P. Fisher, and R.A. Lewis, Phys. Rev. B 61, 7466 (2000).

${ }^{21}$ D. Bimberg, Phys. Rev. B 18, 1794 (1978).

${ }^{22}$ R. A. Lewis and M. Henini, Phys. Status Solidi B 210, 821 (1998). 